Check for updates

Cite this: RSC Adv., 2017, 7, 26130

Received 3rd January 2017

Accepted 1st May 2017

DOI: $10.1039 / \mathrm{c} 7 \mathrm{ra00058h}$

rsc.li/rsc-advances

\section{Point-defect-induced colossal dielectric behavior in GaAs single crystals}

\author{
M. Zhu, (D) a N. Zhang, ${ }^{a}$ H. Wang, ${ }^{a}$ Y. D. Li, ${ }^{a}$ S. G. Huang, ${ }^{a}$ Q. J. Li, ${ }^{\text {*a }}$ Y. Yu, ${ }^{a}$ Y. M. Guo, ${ }^{a}$ \\ X. L. Liu (D) ${ }^{\text {b }}$ and C. C. Wang ${ }^{\star a}$
}

We herein reported colossal dielectric constant (CDC) behavior in GaAs single crystals. This behavior appears in the temperature range above room temperature and results from the bulk effect due to polaron relaxation caused by hopping motion of EL2 defects. When temperature rises higher than $420 \mathrm{~K}$, the interfacial contribution due to Maxwell-Wagner relaxation caused by sample/electrode contacts appears. When temperature is higher than $560 \mathrm{~K}$, the CDC behavior is mainly contributed by the interfacial effect. These features are quite different from the CDC behavior found in oxides, and therefore, the CDC behavior in GaAs single crystals is considered as a new type of the CDC family. Our results underscore the role of point-defects in CDC behavior and suggest that defect engineering can be a promising strategy to achieve superior CDC behavior in both oxide and non-oxide materials.

\section{Introduction}

The continual tendency toward device miniaturization and increasing perceived demand for high-density energy storage have triggered a burst of research activities in searching for colossal permittivity materials..$^{1,2}$ Permittivity over $10^{4}$, is now termed as colossal dielectric constant (CDC). ${ }^{3,4}$ The current existing CDC materials can be classified into two types: (1) ferroelectric materials as represented by $\mathrm{Ba} / \mathrm{Pb}$-based perovskite oxides characterized by a sharp permittivity at the temperature of ferroelectric phase transition, ${ }^{5}$ and (2) non-ferroelectric materials varying from single to multicomponent oxides characterized by a colossal and flat dielectric constant over a wide temperature range around room temperature. ${ }^{6-9}$ Nevertheless, the former suffers from the toxicity and strong temperature dependence of the permittivity; the latter shows the weakness of relatively high dielectric loss. These formidable problems strongly limit the practical application of both types of materials. Therefore, much work is warranted on the searching for new CDC materials suitable for real device application.

Since CDC behavior in non-ferroelectrics and relaxors is contributed exclusively by defect dipolar and/or space charge polarizations, the empirical methods to explore new CDC materials are (1) introducing defect dipoles through doping ${ }^{\mathbf{1 , 1 0 , 1 1}}$ and (2) engineering the space charge polarization by fabricating various artificial interfaces such as core-shell ${ }^{12}$ and hybrid nanolaminate structures..$^{13}$ It is worth emphasizing that all the

${ }^{a}$ Laboratory of Dielectric Functional Materials, School of Physics \& Material Science, Anhui University, Hefei 230601, China. E-mail: liqj@mail.ustc.edu.cn; ccwang@ ahu.edu.cn; Fax: +86 0551 65846849; Tel: +86551 63861902

${ }^{b}$ Chinese Acad Sci, Wuhan Inst Phys \& Math, State Key Lab Magnet Resonance \& Atom $\&$ Mol Phys, Wuhan 430071, China efforts were performed in oxides. In contract, CDC behavior in non-oxide materials remains conspicuously absent. It raises the question: is CDC behavior peculiar to oxides? ${ }^{14}$ Or in other word, does the CDC behavior appear in non-oxide materials?

In fact, point defects and artificial interfaces were widely engineered to tune the properties of non-oxides. ${ }^{15,16}$ It is, therefore, expected that non-oxides could be yet another promising CDC materials. Excited by this expectation, we performed dielectric investigations on GaAs crystals. The sample is used because it has recently gained a revived interest as replacement to silicon in future metal-oxide-semiconductor field-effect transistors. ${ }^{17,18}$ Point defects, such as oxygen, $\mathrm{As}_{\mathrm{Ga}}$ antisite, $\mathrm{Ga} /$ As vacancies, and the complex of these defects, are known to play an important role in determining the properties of GaAs. ${ }^{19-22}$ Our results show that GaAs crystals exhibit CDC behavior in the temperature above room temperature. This behavior is argued to be a bulk effect caused by defect points.

\section{Experimental}

The (001) oriented GaAs single crystals were purchased from Hefei Kejing Materials Technology Co., Ltd. Dielectric properties in both temperature and frequency domains were measured using a Wayne Kerr 6500B precise impedance analyzer (Wayne Kerr Electronic Instrument Co., Shenzhen, China) with the sample mounted in a holder placed inside a PST-2000HL dielectric measuring system (Partulab Co., Wuhan, China). The temperature domain dielectric properties were measured at fixed frequencies by varying temperature with a heating ramp of $2 \mathrm{~K} \mathrm{~min}^{-1}$. The frequency domain dielectric properties were measured at fixed temperatures by scanning frequency from $10^{2}$ to $10^{6} \mathrm{~Hz}$. The ac measuring signal was $100 \mathrm{mV}$ rms. Electrodes 
were made by printing silver paste on both sides of the samples and then fired at $1073 \mathrm{~K}$ for $2 \mathrm{~h}$ in air in order to remove the polymeric component and get good contact. To get detailed information about the dielectric properties of GaAs single crystals, the dielectric data were analyzed in terms of three dielectric functions: dielectric permittivity $\varepsilon^{*}$, electric modulus $M^{*}$, and impedance $Z^{*}$. Electron spin resonance (ESR) measurement was carried out using a Bruker EMX-plus model spectrometer operating at X-band frequency $(v \cong 9.4 \mathrm{GHz})$.

\section{Result and discussion}

Fig. 1(a) and (b) shows, respectively, the temperature (T) dependence of the dielectric constant $\left(\varepsilon^{\prime}\right)$ and loss tangent $(\tan \delta$ ) for a GaAs crystal measured under different frequencies $(f)$. It is seen that a stepwise increase in $\varepsilon^{\prime}(T)$ leads to a colossal and weak temperature-dependent dielectric constant over $4 \mathrm{k}$ around $400 \mathrm{~K}$ of the crystal. The stepwise increase is accompanied by a thermally activated relaxation peak in $\tan \delta$ characterizing the typical the CDC behavior as reported in oxides. ${ }^{2,8}$ However, compared with the CDC behavior in oxides, there are two main features worth to be emphasized: (1) the temperature of the stepwise increase in $\varepsilon^{\prime}$ for the present sample, e.g., $350 \mathrm{~K}$ for the curve recorded at $1 \mathrm{kHz}$, is much higher than those reported in oxides. For example, the stepwise increase temperature recorded with the same frequency of $1 \mathrm{kHz}$ were found to be around $100,150,175$, and $200 \mathrm{~K}$ for $\mathrm{CaCu}_{3} \mathrm{Ti}_{4} \mathrm{O}_{12},{ }^{23} \mathrm{TbMnO}_{3},{ }^{8}$ $\mathrm{LaFeO}_{3},{ }^{24}$ and $\mathrm{La}_{2} \mathrm{CuTiO}_{6}{ }^{25}$ respectively. (2) The stepwise increase in oxides is almost invisible in the curves measured with frequencies higher than $1 \mathrm{MHz}$. Whereas the present sample still shows a stepwise increase larger than $4 \mathrm{k}$ for the curve measured under $3 \mathrm{MHz}$. These features indicate that the CDC behavior in GaAs is different from that in oxides.

To decipher the nature of the CDC behavior in GaAs, knowledge of the mechanism of the observed relaxation is of paramount importance. In doing so, one needs to calculate the relaxation parameters. Nevertheless, a careful examination

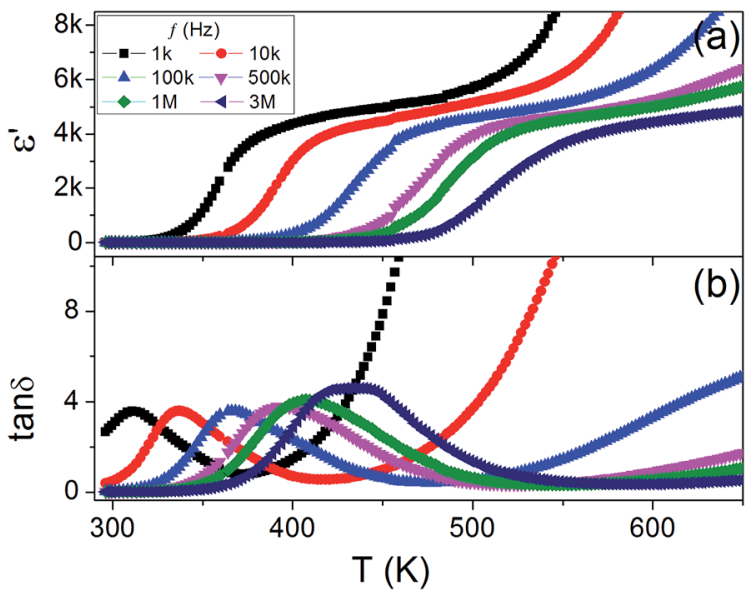

Fig. 1 Temperature dependence of the dielectric constant (a) and loss tangent (b) for a GaAs single crystal measured under different frequencies. reveals that the relaxation peak, especially for those measured with high frequency, e.g., $3 \mathrm{MHz}$, is composed of two close peaks. Meanwhile, an exponentially increasing background in loss tangent at higher temperatures is observed. This background shifts to high temperature and decreases in magnitude with increasing measurement frequency indicating that it is associated with the conductivity [ $\tan \delta \sim \sigma / \omega \sim \exp \left(-E_{\text {cond }} / k_{\mathrm{B}} T\right) /$ $\omega$, where $E_{\text {cond }}$ is activation energy of conductivity, $\omega=2 \pi f$ is angular frequency, and $k_{\mathrm{B}}$ is Boltzmann constant]. Both facts strongly limit us in obtaining the relaxation parameters. In this case, the dielectric function of electric modulus $M^{*}$, defined as $M^{*}=M^{\prime}+j M^{\prime \prime}=1 / \varepsilon^{*}(j$ is the square root of -1$)$, is applied. It shares the same mechanism of the dielectric permittivity $\left(\varepsilon^{*}\right)$ and considered to be a powerful function in revealing the relaxation shadowed by background. ${ }^{26}$

Fig. 2(a) shows the spectroscopic plots of the imaginary part of the electric modulus for a GaAs crystal recorded at a series of temperatures. Owing to the absence of background, two distinct relaxation peaks can be clearly seen. For brevity, these relaxations are named as R1 and R2 in the order of ascending temperature. The peak position, $f_{\mathrm{P}}$, is plotted as a function of the reciprocal of the measuring temperature $(1000 / T)$ in terms of the Arrhenius law

$$
f_{\mathrm{P}}=f_{0} \exp \left(-E_{\mathrm{a}} / k_{\mathrm{B}} T\right)
$$

where $f_{0}$ is pre-exponential factor and $E_{\mathrm{a}}$ is activation energy of the relaxation. Fig. 2(b) displays Arrhenius plots of the observed relaxations. It is seen that both relaxations follow perfect Arrhenius relation. The activation energy is calculated to be 0.80 and $0.48 \mathrm{eV}$ for R1 and R2, respectively. The activation energy of R1 agrees perfectly with that of the EL2 defects $(0.80 \mathrm{eV}) .^{22}$ EL2 defects in GaAs has been generally accepted to be associated with As antisite $\left(\mathrm{As}_{\mathrm{Ga}}\right)$ point defects, ${ }^{27,28}$ which are known to be double donors. The singly charged state $\mathrm{As}_{\mathrm{Ga}}{ }^{+}$has unpaired sp electrons and hence can be detected by ESR spectrum. The ESR result recorded at room temperature is shown in the inset of Fig. 2(b), which shows a resonant line resulting from the $\mathrm{As}_{\mathrm{Ga}}{ }^{+}{ }^{+2}$ This result confirms the existence of the As antisite point defects. On the other hand, the charged defects in the crystal appear to be polarons, because they will be frozen at low enough temperatures by their surrounding medium via repelling (attracting) the like (opposite) ions. At high enough temperatures, these defects will be thermally activated and hop randomly to nearby positions leading to dipolar effect. ${ }^{30}$ Under an applied field, the polarons are electrically activated and hop with respect to the applied field yielding re-orientation polaron relaxation..$^{31}$ Therefore, the relaxation R1 is point-defect related. For a polaron relaxation, the loss tangent and electric modulus attain their maxima of $(\tan \delta)_{\max }$ and $\left(M^{\prime \prime}\right)_{\max }$ at the frequency $f_{\tan \delta}$ and $f_{\mathrm{M}}$, respectively. Based on these parameters, two Arrhenius-like plots are predicated ${ }^{32,33}$

$$
\begin{gathered}
\frac{\left(f_{\tan \delta}\right)^{2}}{f_{\mathrm{M}}} \propto \exp \left(-\frac{W_{\mathrm{H}}}{k_{\mathrm{B}} T}\right) \\
T \frac{(\tan \delta)_{\max }^{2}}{\left(M^{\prime \prime}\right)_{\max }} \propto \exp \left(-\frac{E_{\mathrm{g}}}{2 k_{\mathrm{B}} T}\right)
\end{gathered}
$$



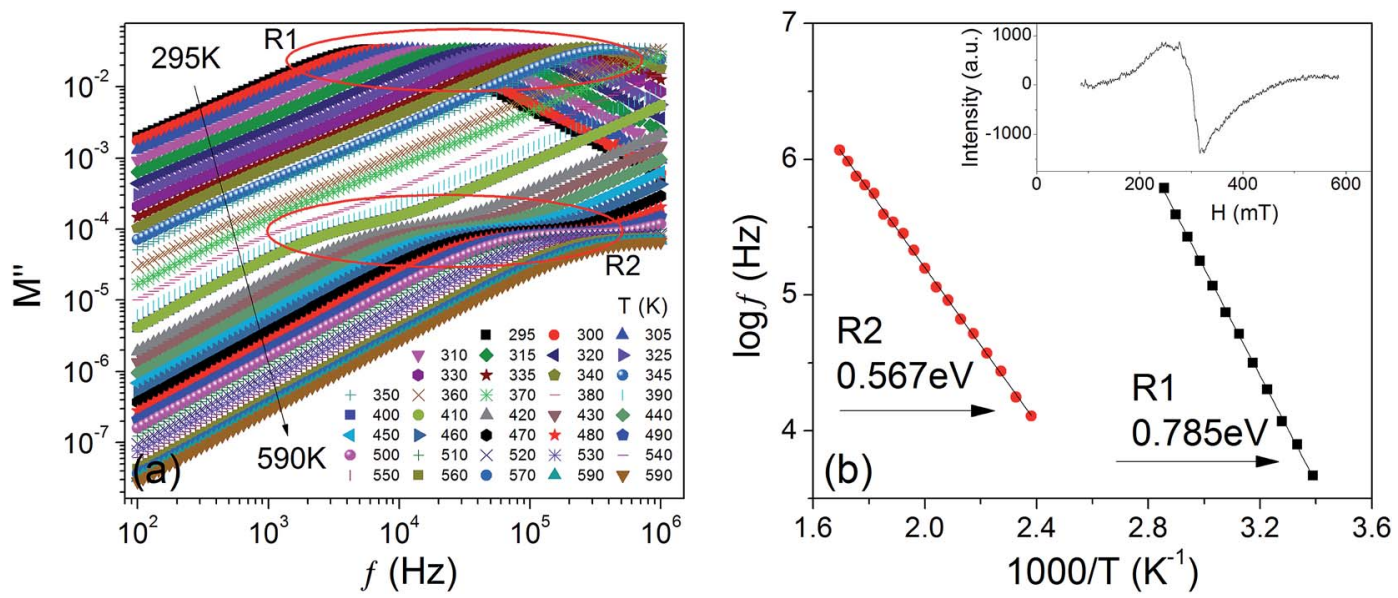

Fig. 2 (a) Frequency dependence of electric modulus for GaAs crystal measures at various temperatures. (b) Arrhenius plots of the relaxations observed in (a). The solid lines are linear fitting results. Inset: ESR spectrum of GaAs crystal recorded at room temperature.

where $W_{\mathrm{H}}$ and $E_{\mathrm{g}}$ are related to polaron hopping and formation energy, respectively. Eqn (2) and (3) predicate a fingerprint nature for a polaron relaxation, namely two Arrhenius-like lines in the plots of $\log \left(\left(f_{\tan \delta}\right)^{2} / f_{\mathrm{M}}\right)$ and $\log \left(T(\tan \delta)_{\max }^{2} /\left(M^{\prime \prime}\right)_{\max }\right)$ against $1 / T$. To corroborate the polaronic nature of R1, the spectroscopic plots of the loss tangent at a series of temperatures are displayed in Fig. 3, from which the maxima of $(\tan \delta)_{\max }$ as well as the position $f_{\tan \delta}$ can be deduced. Together with the spectroscopic plots of electric modulus shown in Fig. 2(a), the two Arrhenius-like relationships are clearly evidenced as shown in the inset of Fig. 3. Linear fittings, shown as solid lines in the inset, yield $W_{\mathrm{H}}=0.750 \mathrm{eV}$ and $E_{\mathrm{g}} / 2=0.052 \mathrm{eV}$. In the case of small polaron hopping process, the activation energy for the conduction follows $E_{\text {con }}=W_{\mathrm{H}}+E_{\mathrm{g}} / 2 .{ }^{33,34}$ The sum of $W_{\mathrm{H}}$ and $E_{\mathrm{g}} / 2$ equals $0.802 \mathrm{eV}$, which agrees perfectly with the activation energy of R1. Since the hopping process of small polaron not only yields bulk conduction but also gives rise to

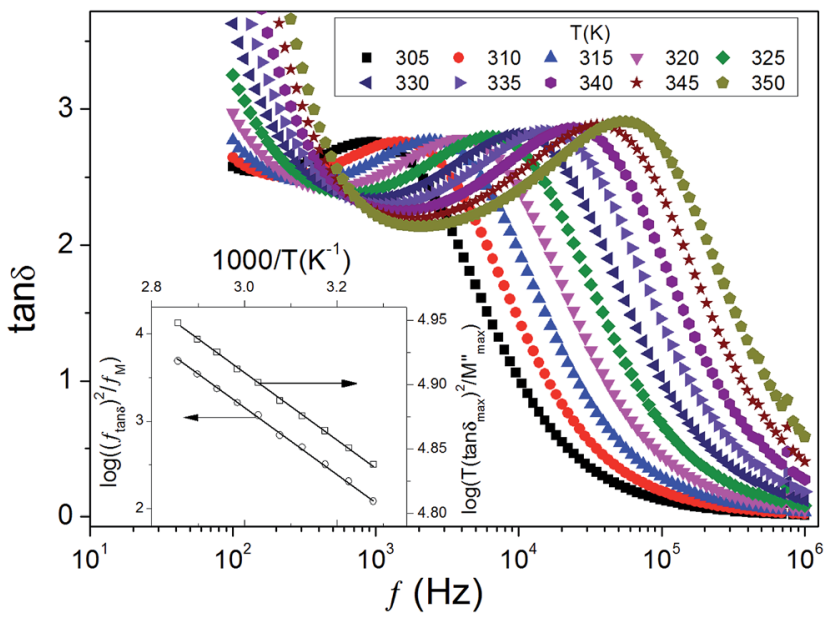

Fig. 3 Frequency dependence of loss tangent of GaAs crystal measures at various temperatures. The inset shows the Arrhenius relations $\left(f_{\tan \delta} \delta\right)^{2} / f_{M}$ and $T(\tan \delta)_{\max }{ }^{2} / M^{\prime \prime}{ }_{\max }$ versus $1000 / T$. The solid lines are linear fitting results. dielectric relaxation, both aspects are strongly related and thus show close value of activation energy. This result firmly confirms the polaronic nature of the R1.

To clarify the nature of R2, we performed impedance analysis under different dc biases at temperatures where $\mathrm{R} 2$ is the predominant relaxation of GaAs. A typical result recorded at 450 $\mathrm{K}$ is present in Fig. 4. This temperature is chosen, because at this temperature only R2 can be seen and R1 moves to a higher frequency out of the measuring frequency window [see Fig. 2(a)]. It is clearly seen that the complex impedance plot behaves as a semicircle indicative of R2. The inset shows an enlarged view of the impedance plot in the high frequency range. It shows that a high-frequency tail independent of dc bias indicative of R1 is almost invisible. We would like to stress that the semicircle can be notably depressed by the dc bias. This finding demonstrates that R2 is an interfacial relaxation or

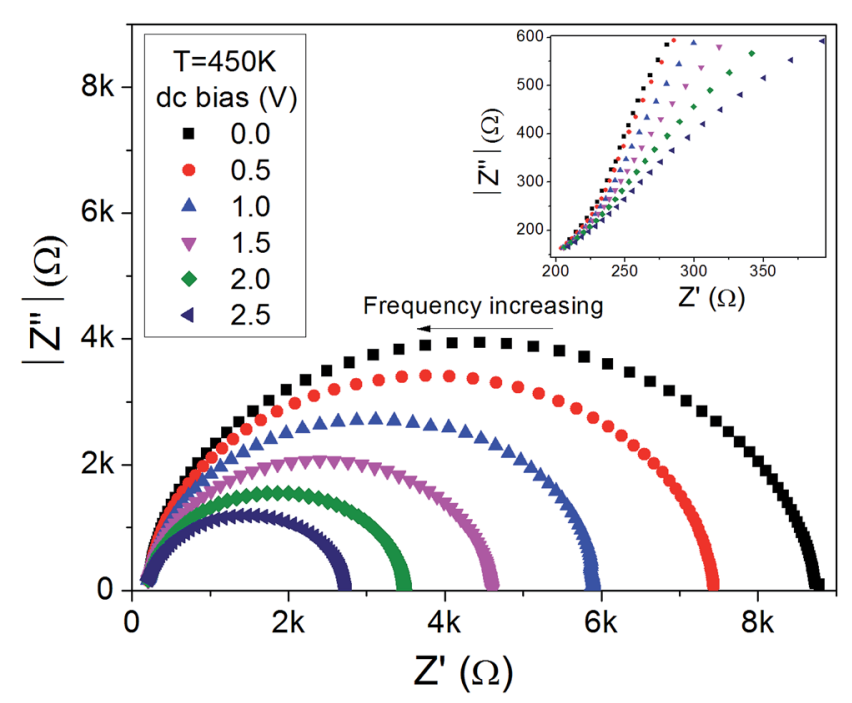

Fig. 4 Complex impedance plots of GaAs crystal recorded at $450 \mathrm{~K}$ under different dc biases. The inset show is an enlarged view of the plots in the high-frequency range. 
Maxwell-Wagner relaxation, because a Maxwell-Wagner relaxation is associated with a Schottky barrier, whose intensity as well as the Maxwell-Wagner relaxation can be effectively depressed by a dc bias. ${ }^{35}$ The absence of grain boundaries in the present crystal leads to the conclusion that R2 is a MaxwellWagner relaxation associated with the interfaces of sample/ electrode contacts.

To further convince this point, we conduct ac conductivity analysis. Fig. 5(a) shows the ac conductivity as a function of frequency recorded at different temperatures. The noteworthy observation is that the curves can be clarified into two regions (I and II). In region I, the curves behave as plateaus in the lowfrequency range followed by linear increases (in log-log scale) in the high-frequency range. This behavior seems to be the typical feature of the well-known universal dielectric response (UDR) as described by ${ }^{36}$

$$
\sigma(T, f)=\sigma_{\mathrm{dc}}+B(T) f^{s}
$$

where $\sigma_{\mathrm{dc}}$ is dc conductivity, $B(T)$ and $s$ are temperaturedependent constants. However, a careful examination reveals that the linear increase is actually a sigmoidal curve which collects the low-frequency $\sigma_{\mathrm{dc}}$ and a high-frequency plateau. The high-frequency plateau is more notable in the curves measured at low temperatures, e.g., $320 \mathrm{~K}$. This fact indicates that the UDR model fails to describe the ac conductivity. Since both the Maxwell-Wagner and polaron relaxations have contributions to the ac conductivity, in this case, the sample can be modeled by two serially connected $R C$ ( $R=$ resistor and $C=$ capacitor) units as shown by the inset in Fig. 5(b) where the subindex 1 and 2 refer to the bulk and interfacial effects, respectively. Based on the equivalent circuit, the resistance of the system can be written as $^{37}$

$$
R=\frac{\left(R_{1}+R_{2}\right)^{2}+\omega^{2} R_{1}{ }^{2} R_{2}^{2}\left(C_{1}+C_{2}\right)^{2}}{R_{1}+R_{2}+\omega^{2} R_{1} R_{2}\left(R_{1} C_{1}{ }^{2}+R_{2} C_{2}{ }^{2}\right)}
$$

where $\omega=2 \pi f$ is the angular frequency. By considering the behavior at zero and infinite frequencies and introducing the parameters of $A=$ area of the capacitor and $l=$ sample thickness, eqn (5) can be rewritten as

$$
\sigma(\omega)=\sigma_{\infty}+\frac{\sigma_{0}-\sigma_{\infty}}{1+(\omega \tau)^{2}}
$$

with $\quad \sigma_{0}(\omega=0)=\frac{l}{A} \frac{1}{R_{1}+R_{2}}, \quad \sigma_{\infty}(\omega \rightarrow \infty)=\frac{l}{A} \frac{R_{1} C_{1}{ }^{2}+R_{2} C_{2}{ }^{2}}{R_{1} R_{2}\left(C_{1}+C_{2}\right)^{2}}$, and $\tau=\frac{R_{1} R_{2}\left(C_{1}+C_{2}\right)}{R_{1}+R_{2}}$.

Similar to Debye relaxation, eqn (6) clearly indicates that the ac conductivity shows a low- and high-frequency plateaus with a sigmoidal curve linking them. It is generally accepted that $R_{2}$ $\gg R_{1}, C_{2} \gg C_{1} \cdot{ }^{38}$ As a result, $\sigma_{0} \sim 1 / A R_{2}$ and $\sigma_{\infty} \sim 1 / A R_{1}$. This means that the low- and high-frequency plateaus represent the interfacial and bulk contributions, respectively. It, therefore, follows that the region I in Fig. 5(a) represents the interfacial effect. The Arrhenius plot of the dc conductivity deduced from region I of Fig. 5(a) is plotted in Fig. 5(b). The activation energy is calculated to be $0.51 \mathrm{eV}$, which is comparable with that R2. This finding confirms that the $\mathrm{R} 2$ is caused by interfacial effect.

The above results evidence that $\mathrm{R} 1$ and $\mathrm{R} 2$ are related to bulk and interfacial effects due to polaron and Maxwell-Wagner relaxations, respectively. In order to find out which one dominates the CDC behavior of the GaAs crystal, the frequency dependence of the real part of the complex permittivity was measured at temperatures varying from 300 to $600 \mathrm{~K}$ with an increment step of $20 \mathrm{~K}$. As shown in Fig. 6, the CDC behavior of GaAs results from two thermally activated relaxations. The relaxation R1 characterized by a low-frequency plateau and a stepwise decrease in $\varepsilon^{\prime}(f)$ dominates the CDC behavior in the temperature range of $T \leq 540 \mathrm{~K}$. When $T \geq 420 \mathrm{~K}$, though the data points at the lowest frequencies deviate from the lowfrequency plateau indicative of the appearance of R2, only when $T \geq 560 \mathrm{~K}$, the contribution to the CDC behavior from R2 outmatches that from R1 (please note the logarithmic scale of the dielectric constant). Therefore, the observed CDC behavior in GaAs crystal is dominated by the bulk effect due to polaron relaxation caused by the hopping motion of EL2 defects in the temperature range below $560 \mathrm{~K}$. While in the temperature range

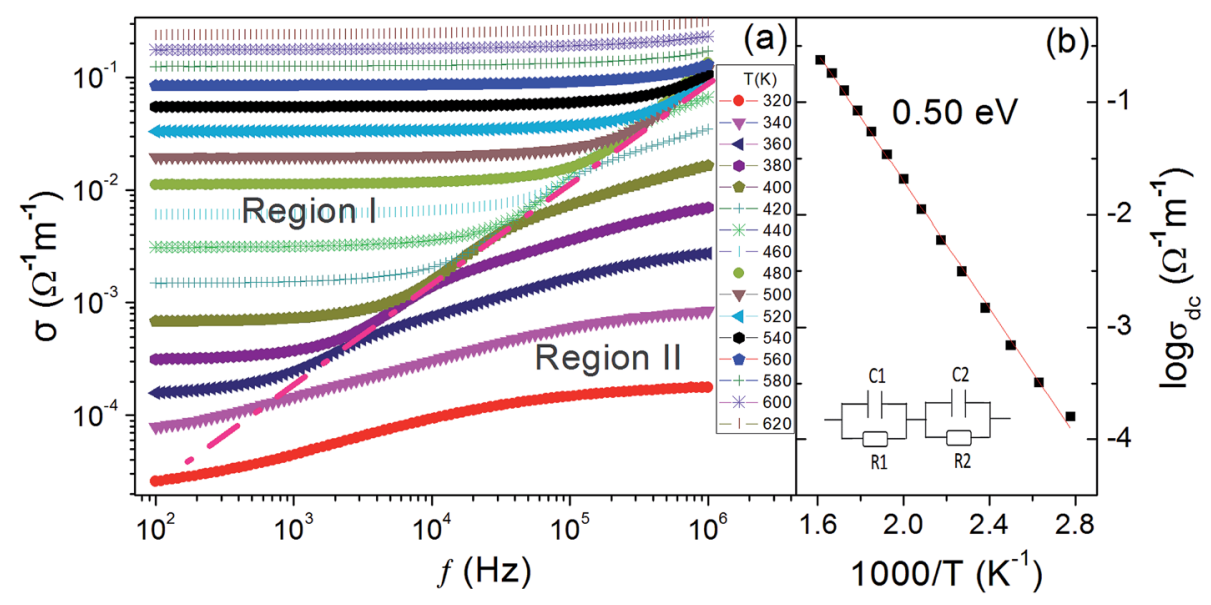

Fig. 5 (a) Frequency dependence of ac conductivity of GaAs crystal measured at various temperatures. (b) Arrhenius plot of the dc conductivity deduced from (a). The solid line is linear fitting result and the inset is the equivalent circuit. 


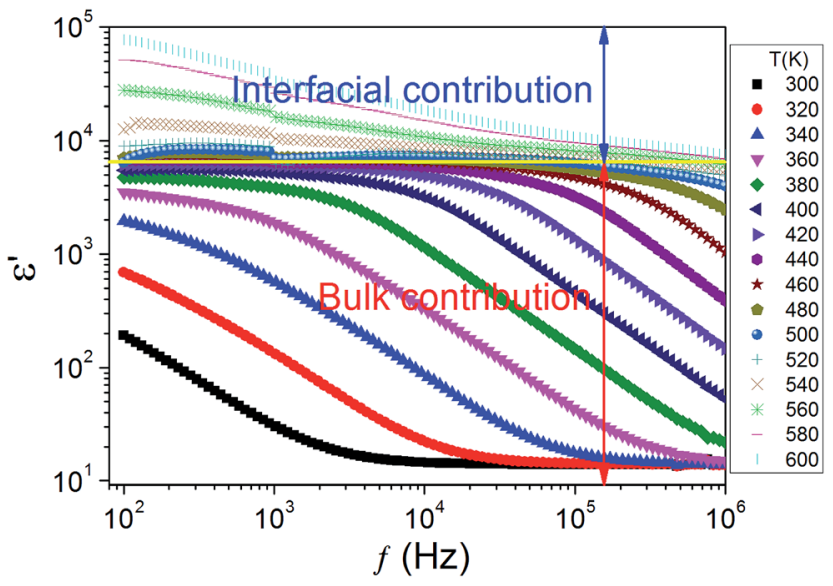

Fig. 6 Frequency dependence of the dielectric constant for GaAs crystal measured at various temperatures.

above $560 \mathrm{~K}$, the CDC behavior is mainly contributed by the interfacial effect due to Maxwell-Wagner relaxation caused by the sample/electrode contacts. This behavior is quite different from that observed in $\mathrm{CaCu}_{3} \mathrm{Ti}_{4} \mathrm{O}_{12}$. The interfacial effect therein contributes $\sim 3 / 4$ CDC behavior of $\mathrm{CaCu}_{3} \mathrm{Ti}_{4} \mathrm{O}_{12} \cdot{ }^{39}$ Therefore, the CDC behavior in GaAs crystals can be considered as new type one.

\section{Conclusions}

In conclusion, dielectric properties of GaAs crystals were investigated in the temperature from room temperature to 650 $\mathrm{K}$ and frequency range of $10^{2}$ to $3 \times 10^{6} \mathrm{~Hz}$. The crystal shows CDC behavior contributed by bulk and interfacial effects in the temperature ranges below and above $560 \mathrm{~K}$, respectively. The bulk contribution comes from the polaron relaxation caused by the hopping motion of EL2 defects. Whereas the interfacial contribution results form the Maxwell-Wagner relaxation caused by the sample/electrode contacts. The CDC behavior in GaAs crystals is argued to be a new one different form the conventional type reported in oxides.

\section{Acknowledgements}

The authors thank financial support from National Natural Science Foundation of China (Grant No. 51572001, 11404002 and 11404003). This work was supported in part by the Weak Signal-Detecting Materials and Devices Integration of Anhui University (Grant No. Y01008411).

\section{References}

1 W. B. Hu, Y. Liu, R. L. Withers, T. J. Frankcombe, L. Noren, A. Snashall, M. Kitchin, P. Smith, B. Gong, H. Chen, J. Schiemer, F. Brink and J. Wong-Leung, Nat. Mater., 2013, 12, 821-826.

2 C. C. Homes, T. Vogt, S. M. Shapiro, S. Wakimoto and A. P. Ramirez, Science, 2001, 293, 673-676.
3 P. Lunkenheimer, V. Bobnar, A. V. Pronin, A. I. Ritus, A. A. Volkov and A. Loidl, Phys. Rev. B: Condens. Matter Mater. Phys., 2002, 66, 052105.

4 P. Lunkenheimer, R. Fichtl, S. G. Ebbinghaus and A. Loidl, Phys. Rev. B: Condens. Matter Mater. Phys., 2004, 70, 172102.

5 L. E. Cross, Ferroelectrics, 1987, 76, 241-267.

6 P. H. Salame, O. Prakash and A. R. Kulkarni, Ceram. Int., 2016, 42, 13207-13214.

7 W. Q. Cao, L. F. Xu, M. M. Ismail and L. L. Huang, Mater. Sci., 2016, 34, 322-329.

8 C. C. Wang, Y. M. Cui and L. W. Zhang, Appl. Phys. Lett., 2007, 90, 012904

9 D. Berardan, S. Franger, D. Dragoe, A. K. Meena and N. Dragoe, Phys. Status Solidi RRL, 2016, 10, 328-333.

10 J. B. Wu, C. W. Nan, Y. H. Lin and Y. Deng, Phys. Rev. Lett., 2002, 89, 217601.

11 X. Liu, H. Q. Fan, J. Shi and Q. Li, Sci. Rep., 2015, 5, 1269912709.

12 X. Y. Zhang, Y. J. Chen, Z. Y. Li and V. G. Harris, J. Alloys Compd., 2017, 692, 950-954.

13 W. Li, O. Auciello, R. N. Premnath and B. Kabius, Appl. Phys. Lett., 2010, 96, 162907.

14 N. Izyumskaya, Y. Alivov and H. Morkoc, Crit. Rev. Solid State Mater. Sci., 2009, 34, 89-179.

15 M. G. Kibria, F. A. Chowdhury, S. Zhao, M. L. Trudeau, H. Guo and Z. Mi, Appl. Phys. Lett., 2015, 106, 113105.

16 Z. C. Zhang, B. Xu and X. Wang, Chem. Soc. Rev., 2014, 43, 7870-7886.

17 J. A. Del Alamo, Nature, 2011, 479, 317-323.

18 J. Nah, H. Fang, C. Wang, K. Takei, M. H. Lee, E. Plis, S. Krishna and A. Javey, Nano Lett., 2012, 12, 3592-3594.

19 D. Colleoni and A. Pasquarello, Phys. Rev. B, 2016, 93, 125208.

20 H. B. Shu, X. D. Yang, P. Liang, D. Cao and X. S. Chen, J. Phys. Chem. C, 2016, 120, 22088-22095.

21 V. Saltas, A. Chroneos and F. Vallianatos, RSC Adv., 2016, 6, 53324-53330.

22 S. T. Neild, M. Skowronski and J. Lagowski, Appl. Phys. Lett., 1991, 58, 859-861.

23 C. C. Wang and L. W. Zhang, Appl. Phys. Lett., 2007, 90, 142905.

24 C. Chen, K. B. Xu, Y. M. Cui and C. C. Wang, Mater. Lett., 2012, 89, 153-155.

25 W. Z. Yang, M. M. Mao, X. Q. Liu and X. M. Chen, J. Appl. Phys., 2010, 107, 124102.

26 C. C. Wang, J. Wang, X. H. Sun, L. N. Liu, J. Zhang, J. Zheng and C. Cheng, Solid State Commun., 2014, 179, 29-33.

27 X. F. Zhong, D. S. Jiang, W. Ge and C. Song, Appl. Phys. Lett., 1988, 52, 628-630.

28 S. M. Tunhuma, F. D. Auret, M. J. Legodi and M. Diale, J. Appl. Phys., 2016, 119, 145705.

29 D. W. Jung, J. P. Noh and N. Otsuka, Phys. B, 2010, 405, 41334138.

30 C. C. Wang and L. W. Zhang, Appl. Phys. Lett., 2008, 92, 132903.

31 A. K. Jonscher, Nature, 1975, 253, 717-719. 
32 G. J. Wang, C. C. Wang, S. G. Huang, C. M. Lei, X. H. Sun, T. Li and L. N. Liu, J. Am. Ceram. Soc., 2013, 96, 2203-2210. 33 E. Iguchi, K. Ueda and W. H. Jung, Phys. Rev. B: Condens. Matter Mater. Phys., 1996, 54, 17431-17437.

34 W. H. Jung, J. Mater. Sci. Lett., 2002, 21, 573-575.

35 C. C. Wang, M. He, F. Yang, J. Wen, G. Z. Liu and H. B. Lu, Appl. Phys. Lett., 2007, 90, 192904.

36 A. K. Jonscher, Nature, 1997, 267, 673-679.
37 R. Stumpe, D. Wagner and D. Bauerle, Phys. Status Solidi A, 1983, 75, 143-154.

38 P. Lunkenheimer, S. Krohns, S. Riegg, S. G. Ebbinghaus, A. Reller and A. Loidl, Eur. Phys. J.: Spec. Top., 2010, 180, 61-89.

39 C. C. Wang, W. Ni, D. Zhang, X. Sun, J. Wang, H. B. Li and N. Zhang, J. Electroceram., 2016, 36, 46-57. 